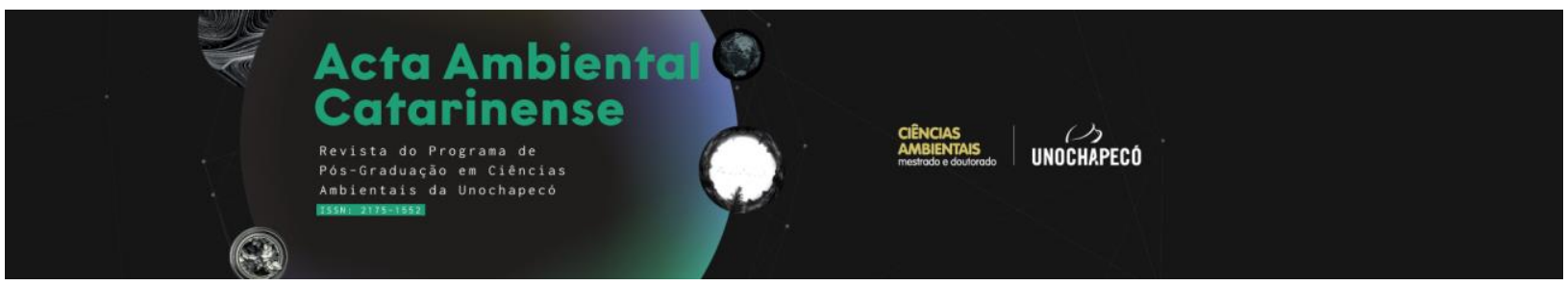

\title{
TRATAMENTO AERÓBIO DE ESGOTO EMPREGANDO A TÉCNICA DE BIOAUMENTAÇÃO
}

\author{
Guilherme Pereira de Matos ${ }^{1}$ \\ Jéssica Talita Zagonel ${ }^{2}$
}

\begin{abstract}
Resumo
Quando descartado sem tratamento, o esgoto pode causar impactos negativos no meio ambiente, tornando, assim, seu tratamento imprescindível. E dentre as técnicas disponíveis, a bioaumentação vem provando ser um método valioso na remoção de diversos poluentes. Perante o exposto, o presente estudo investigou o emprego da bioaumentação no tratamento do esgoto proveniente de uma universidade. Os experimentos transcorreram inicialmente, com a adição de $1 \mathrm{~mL}$ de esgoto bruto em frascos contendo 4, 9, 19 e $29 \mathrm{~mL}$ de caldo nutriente, respectivamente. Posterior ao período de incubação, incorporaram-se estes frascos a $2 \mathrm{~L}$ de esgoto bruto que foram aerados por $240 \mathrm{~h}$. Amostras foram retiradas nos tempos 24, 72, 144, 192 e 240 h e submetidas a análises físicoquímicas. Ao término dos experimentos, chegou-se à conclusão que o recipiente que recebeu $9 \mathrm{~mL}$ de caldo nutriente demonstrou melhores resultados na remoção de cor $(95 \%)$, turbidez (97\%) e DQO $(95,4 \%)$ após 192 h de tratamento, já o frasco controle apresentou, para o mesmo período, remoção de $89,5 \%$ para cor, $95,7 \%$ para turbidez e $94 \%$ para DQO. Os resultados apontam para a eficiência do uso da bioaumentação, porém recomenda-se que análises mais detalhadas sejam realizadas para comprovar a eficiência da técnica.
\end{abstract}

Palavras-chave: Degradação Aeróbia. Biorremediação. Bioaumentação.

\begin{abstract}
When discarded without treatment, sewage can cause negative impacts on the environment, thus making its treatment essential. And among the techniques available, bioaugmentation has been proving to be a valuable method for removing various pollutants. Given the above, the present study investigated the use of bioaugmentation in the treatment of sewage from a university. The experiments were carried out, initially, with the addition of $1 \mathrm{~mL}$ of raw sewage in flasks containing 4, 9, 19 and $29 \mathrm{~mL}$ of nutrient broth, respectively. Subsequently to the incubation period, these flasks were added to $2 \mathrm{~L}$ of raw sewage, which ware aerated for up to $240 \mathrm{~h}$. Samples were taken at times 24, 72, 144, 192 and $240 \mathrm{~h}$ and submitted to physical-chemical analysis. At the end of the experiments, it was concluded that the container that received $9 \mathrm{~mL}$ of nutrient broth showed better

\footnotetext{
${ }^{1}$ Acadêmico do curso de Engenharia Sanitária e Ambiental da Universidade do Oeste de Santa Catarina - Unoesc Videira.

${ }^{2}$ Mestre em Ciência e Biotecnologia pela Universidade do Oeste de Santa Catarina - Unoesc Videira. 
results in removing color (95\%), turbidity (97\%) and COD (95.4\%) after $192 \mathrm{~h}$ of treatment, whereas the control flask, for the same period, showed a removal of $89.5 \%$ for color, $95.7 \%$ for turbidity and $94 \%$ for COD. The results point to the efficiency of the use of bioaugmentation, however it is recommended that more detailed analyzes be performed to prove the efficiency of the technique.

Keywords: Aerobic degradation. Bioremediation. Bioaugmentation.

\section{INTRODUÇÃo}

O esgoto doméstico é constituído, basicamente, por $99,9 \%$ de água e $0,1 \%$ de sólidos, como areia, sólidos em suspensão e dissolvidos (KOREN e BISESI, 2003; NUVOLARI, 2011; TOPARE et al., 2011) e microrganismos (SPERLING, 2002). No total desta composição, é possível detectar, ainda, compostos como: fosfatos, sulfatos, carbonatos, ureia, amônia, ácido úrico e gorduras, além de diversas substâncias eliminadas pela urina e fezes humanas, ou por meio da lavagem de louças e roupas (NUVOLARI, 2011).

Quando descartado sem tratamento em corpos hídricos, o esgoto pode causar, entre outros problemas: a redução das taxas de oxigênio dissolvido, aumento da turbidez (TOPARE et al., 2011; SOUSA, 2016) e da carga de nutrientes (TOPARE et al. 2011; TURCI et al., 2017), emanação de maus odores (SOUSA, 2016) e aumento da quantidade de organismos patogênicos presentes na água (TCHOBANOGLOUS et al., 2013; TURCI et al., 2017).

Em razão da sua periculosidade, o tratamento desta espécie de água residuária é imprescindível. Para tanto, a literatura menciona diferentes formas ou meios de reduzir a carga de poluentes e patógenos dos esgotos, sendo os processos biológicos apontados com uma tecnologia relativamente acessível e cujos produtos finais $\mathrm{da}$ mineralização completa não demonstram características tóxicas.

Dentre os processos de biorremediação, destaca-se a técnica de bioaumentação, que consiste na introdução de uma cepa específica ou de um consórcio de organismos para aumentar a atividade biológica de um sistema (HERRERO e STUCKEY, 2015; SHAH, 2017; SZAJA, 2018) facilitando assim o processo de remediação.

A bioaumentação vem provando ser um método valioso para melhorar $\mathrm{o}$ desempenho no tratamento (ZHANG et al., 2017) e resolver problemas práticos em estações de tratamento de águas (HERRERO e STUCKEY, 2015). Além disto, pode otimizar o catabolismo de compostos específicos (por exemplo, orgânicos refratários ou demanda química de oxigênio DQO geral) (HERRERO e STUCKEY, 2015), melhorar a remoção de contaminantes, reduzir o tempo e os custos (SPEIGHT e EL-GENDY, 2018; SKINDER et al., 2020) do tratamento.

Diante do exposto nos parágrafos anteriores, o presente trabalho tem por objetivo avaliar o emprego da técnica de bioaumentação no tratamento do esgoto oriundo de uma universidade.

\section{MATerial E MÉtodos}

\subsection{Local de estudo}

A água residuária utilizada neste trabalho foi coletada no tanque séptico para onde é destinado o esgoto produzido, em sua grande maioria, pelos sanitários de um dos blocos estudantis que compõem a estrutura do campus da Universidade do Oeste de Santa Catarina - Unoesc Videira. 


\subsection{Preparação do inóculo}

Para a preparação dos inóculos, adicionou-se em cada frasco de vidro 4, 9, 19 e $29 \mathrm{~mL}$ de caldo nutriente, após esterilização, acrescentou-se a estes $1 \mathrm{~mL}$ de esgoto. Os frascos em seguida foram incubados em estufa numa temperatura de $37 \pm 1{ }^{\circ} \mathrm{C}$ por 24 horas.

O caldo nutriente empregado durante a realização dos experimentos apresenta na sua composição $\left(\mathrm{g} \mathrm{L}^{-1}\right): 1,0$ de extrato de bife; 2,0 de extrato de levedura; 5,0 de peptona e 5,0 de cloreto de sódio. A sua preparação deu-se seguindo as instruções contidas no rótulo do meio de cultura.

\subsection{Simulação do tratamento aeróbio}

Em recipientes de vidro contendo 2 litros de esgoto bruto acoplaram-se bombinhas de aquário, que forneciam oxigênio para simular o processo de tratamento aeróbio. A estes mesmos recipientes incorporaram-se os frascos com inóculos, após transcorrido o período de incubação. Ressalta-se que um dos recipientes não recebeu o inóculo, obtendo-se assim o tratamento controle.

O processo de tratamento ocorreu por um período de 240 horas. Este intervalo de tempo foi escolhido com base na literatura disponível a respeito de sistemas de tratamentos biológicos aeróbios. Já que dependendo do modelo de tratamento escolhido, o tempo de detenção pode variar, em média, entre 48 a 240 h (VON SPERLING, 2005).

Além disto, optou-se por realizar os experimentos no período do verão, pois as temperaturas se mantêm em média entre as ideais, 25 a $40{ }^{\circ} \mathrm{C}$, para o ótimo crescimento de microrganismos mesófilos. Segundo Tortora et al. (2017) grande parte dos microrganismos, inclusive os deteriorantes, encaixam-se nesta classe.

\subsection{Análises físico-químicas}

Com o intuito de aferir a eficiência dos tratamentos, amostras foram retiradas nos intervalos de tempo $24,72,144,192$ e $240 \mathrm{~h}$ e submetidas a análises físico-químicas. Os parâmetros avaliados foram: Amônia, Cor (465 nm), Demanda Química de Oxigênio (DQO), Nitrito e Turbidez. Além disto, monitorou-se as taxas de oxigênio e $\mathrm{pH}$ das amostras. Para a análise dos parâmetros físicoquímicos, utilizou-se das metodologias descritas em Standard Methods for the Examination of Water and Wastewater (APHA, 2012).

\section{Resultados E Discussões}

\subsection{Caracterização do esgoto bruto}

$\mathrm{Na}$ Tabela 1 segue exposto o valor médio juntamente com o desvio padrão, observado para cada parâmetro escolhido durante a caracterização inicial da água residuária estudada.

A água residuária avaliada possui $\mathrm{pH}$ médio de 5,91. Tal valor é inferior ao observado por autores como: Von Sperling (2005), cujos estudos apontam para $\mathrm{pH}$ de esgoto variando na faixa de 6,7 -8,0; Turci et al. (2017) que identificaram um valor mínimo de 6,61 e máximo de 7,95; Colares e Sandri (2013) que obtiveram um $\mathrm{pH}$ de 8,23 e Tonetti et al. (2012) que constataram um $\mathrm{pH}$ de 6,9 \pm 0,3 para o esgoto avaliado.

A discrepância entre os resultados anteriormente observados está, possivelmente, relacionada a problemas na digestão anaeróbica, visto que, a maioria das complicações com este processo pode ser atribuída ao acúmulo de ácidos voláteis, que por consequência provocam a queda do $\mathrm{pH}$. A redução do $\mathrm{pH}$ para valores abaixo de 6,6 implica na inibição do crescimento das Arqueas metanogênicas. Por outro lado, as bactérias acidogênicas continuam suas funções até $\mathrm{pH} 4,5$, resultando num rápido 
acúmulo de ácidos orgânicos voláteis (AMARAL et al., 2019) no meio.

As principais fontes de compostos nitrogenados no esgoto bruto são proteínas, aminas, aminoácidos e ureia. $\mathrm{O}$ nitrogênio amoniacal é resultado da decomposição bacteriana destes compostos orgânicos (MANYUCHI et al., 2019). Nas amostras analisadas o teor de amônia ficou dentro da média de 20,77 $\mathrm{mg} \mathrm{L}^{-1}$, apresentando pico de $33 \mathrm{mg} \mathrm{L}^{-1} \mathrm{em}$ alguns dias. Os resultados desta pesquisa condizem com estudos que mostram a concentração de nitrogênio amoniacal no esgoto variando entre $20-35 \mathrm{mg} \mathrm{L}^{-1}$ (VON SPERLING, 2005).

Tabela 1. Caracterização do esgoto bruto.

\begin{tabular}{lc}
\hline \multicolumn{1}{c}{ Parâmetro } & Média \pm DP* \\
\hline Amônia $\left(\mathrm{mg} \mathrm{L}^{-1}\right)$ & $20,77 \pm 10,71$ \\
Cor aparente $\left(\mathrm{mg} \mathrm{Pt} \mathrm{Co} \mathrm{L}^{-1}\right)$ & $2112,22 \pm 779,39$ \\
Cor real $\left(\mathrm{mg} \mathrm{Pt} \mathrm{Co} \mathrm{L}^{-1}\right)$ & $988,28 \pm 44,21$ \\
DQO (mg L & \\
Oxigênio dissolvido & $585,63 \pm 31,05$ \\
$\left(\mathrm{mg} \mathrm{L}^{-1}\right)$ & $2,55 \pm 0,58$ \\
Nitrito $\left(\mathrm{mg} \mathrm{L}^{-1}\right)$ & $0,22 \pm 0,08$ \\
pH & $5,91 \pm 0,17$ \\
Sólidos Suspensos (mg L-1 & $6400,00 \pm$ \\
Turbidez (NTU) & 5122,50 \\
\hline
\end{tabular}

* DP $=$ Desvio Padrão

NTU - Unidade Nefelométrica de Turbidez

$\mathrm{O}$ esgoto analisado apresenta concentração máxima de nitrito de $0,30 \mathrm{mg} \mathrm{L}^{-}$ ${ }^{1}$ e média de $0,22 \mathrm{mg} \mathrm{L}^{-1}$. Os teores médios das amostras são próximos ao constatado por Peixoto et al. (2012) em seus experimentos $\left(0,16 \mathrm{mg} \mathrm{L}^{-1}\right)$. Ademais, o nitrito, por ser instável no esgoto, se oxida facilmente para nitrato, sendo que raramente excede a $1 \mathrm{mg} \mathrm{L}^{-}$ ${ }^{1}$ (JORDÃO e PESSÔA, 2005).

Com relação à DQO, obteve-se uma média de 585,63 $\mathrm{mg} \mathrm{L}^{-1}$ durante os ensaios, tal resultado situa-se dentro dos teores de DQO para esgoto estimado por Jordão e Pessôa
(2005), 200-800 mg L ${ }^{-1}$, Von Sperling (2005), $450-800 \mathrm{mg} \mathrm{L}^{-1}$, e Manyuchi et al. (2019) 200$700 \mathrm{mg} \mathrm{L}^{-1}$.

Por fim, a cor e a turbidez são parâmetros utilizados para indicar o estado de decomposição do esgoto (JORDÃO e PESSÔA, 2005). Segundo Tchobanoglous et al. (2013), tonalidades usualmente marrons acinzentadas claras indicam esgoto fresco. Sendo assim, com base no disposto na literatura, o esgoto analisado é fresco (Figura $1)$.

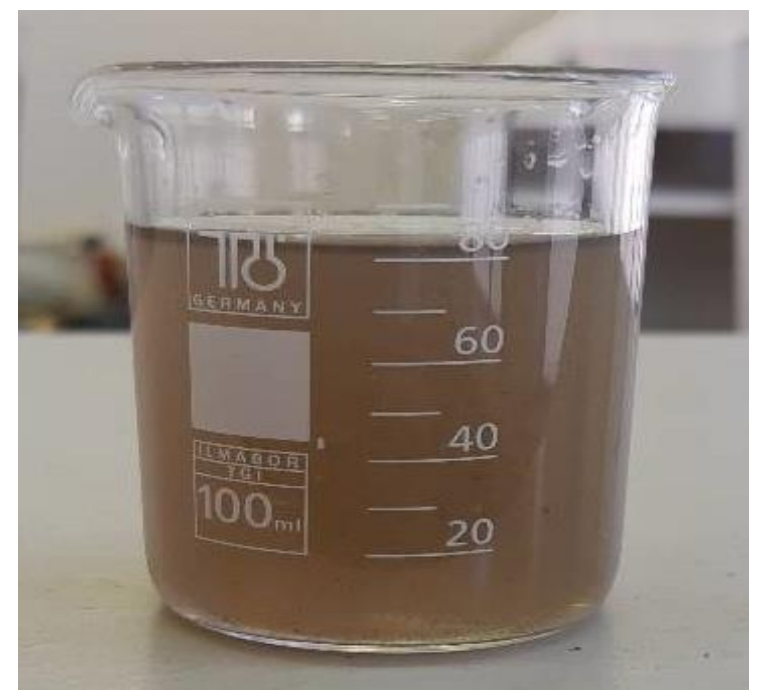

Figura 1. Imagem do Esgoto bruto.

\subsection{Ensaios laboratoriais de biorremediação}

Com o intuito de aferir a eficiência da técnica de bioaumentação na remediação do esgoto analisado, incorporaram-se frascos com caldo nutriente $(4,9,19$ e $29 \mathrm{~mL})$ e colônias de microrganismo à recipientes contendo 2 litros de esgoto, que foram mantidos sob aeração por $240 \mathrm{~h}$.

Optou-se pelo uso do consórcio de microrganismos presentes no esgoto bruto, pois assim problemas de adaptabilidade com o meio a ser degradado e com os microrganismos predando uns aos outros poderiam ser evitados.

Textos da literatura afirmam que a introdução de microrganismos isolados do 
próprio resíduo a ser tratado pode melhorar a degradação de poluentes orgânicos em águas residuárias (HESNAWI et al., 2014). Além disto, quando os microrganismos trabalham em conjunto na forma de consórcio, permitem uma degradação rápida e aprimorada de muitos contaminantes devido, possivelmente, à atividade co-metabólica de uma espécie que complementa a outra (MEHROTRA et al., 2019).

Em análise do Figura 2, é possível afirmar que o processo biológico reduz de forma significativa os teores de cor presente no esgoto, dado que para todas as situações propostas de tratamento houve uma redução maior que $70 \%$ deste parâmetro, a partir das 72 horas.

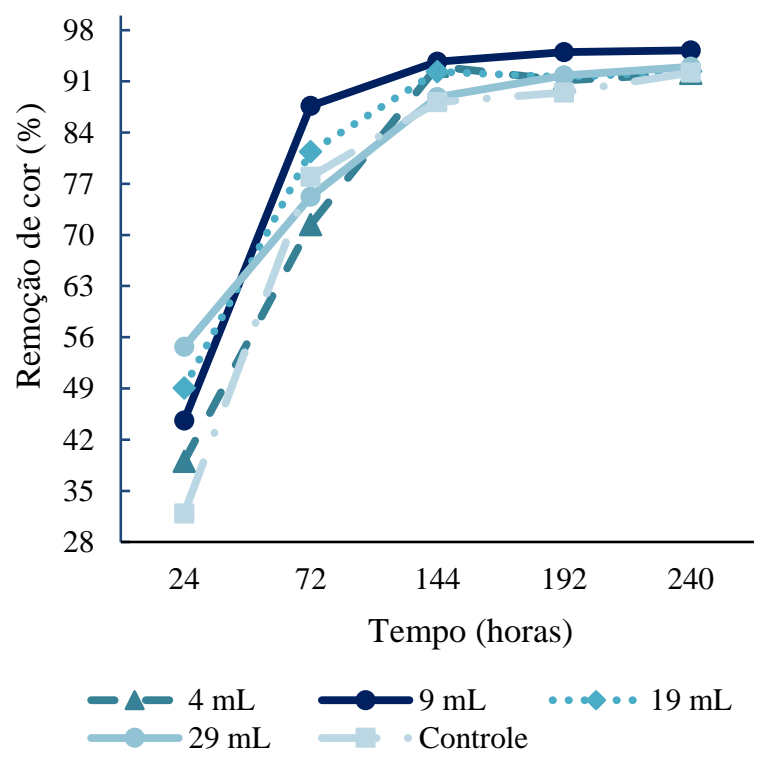

Figura 2. Remoção de cor nos diferentes ensaios de bioaumentação.

Tal fenômeno, provavelmente, esta associado à degradação da matéria orgânica, já que, a cor de uma amostra de água está relacionada à redução que a intensidade da luz sofre ao atravessá-la (NOWACKI e RANGEL, 2014), em razão da presença de sólidos, principalmente, material em estado coloidal orgânico (MANAGO et al., 2018).

Contudo, constatou-se que o recipiente que recebeu $9 \mathrm{~mL}$ de caldo nutriente, dentre todos, demonstrou melhor remoção de cor a partir de 72 horas de tratamento, sendo que índices de maior eficiência foram observados nos tempos 144 h (94\%), 192 h (95\%) e 240 $(95,2 \%)$. O controle, por sua vez, apresentou eficácia de 88,1\% em 144 h, 89,5\% em 192 h e $92,2 \%$ em $240 \mathrm{~h}$.

Igualmente ao que ocorreu na remoção da cor, o frasco em que se adicionou $9 \mathrm{~mL}$ de caldo nutriente manifestou uma maior redução de turbidez (Figura 3), 96,8\% em 144 h e $97 \%$ em $192 \mathrm{~h}$, quando comparado como os demais tratamentos. O controle para o mesmo parâmetro apresentou eficiência de 93,2\% em $144 \mathrm{~h}$ e $95,7 \%$ em $192 \mathrm{~h}$.

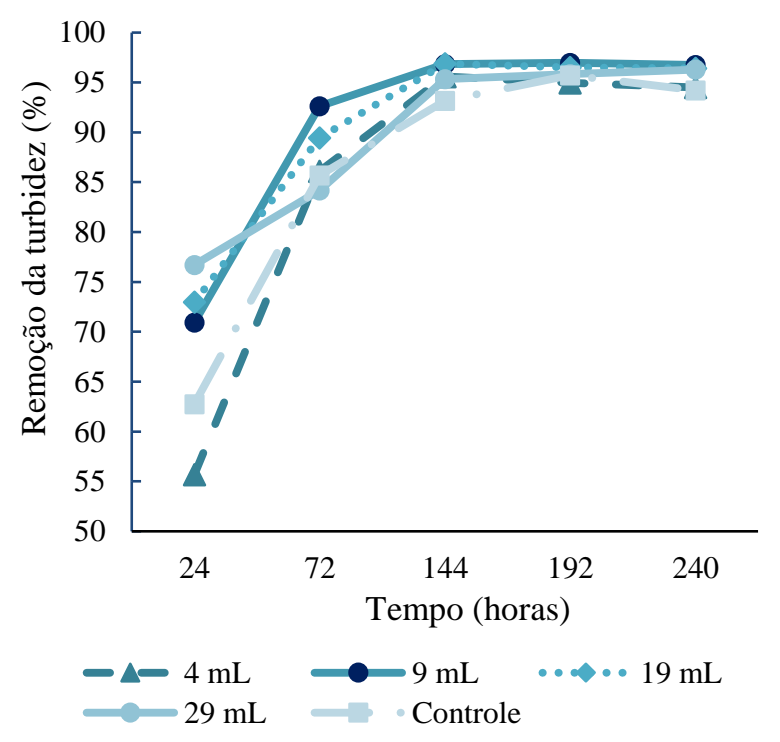

Figura 3. Remoção de turbidez nos diferentes ensaios de bioaumentação.

Com relação à redução da DQO (Figura 4), os frascos onde acrescentou-se 9 e $19 \mathrm{~mL}$ de caldo apresentaram em um período de $144 \mathrm{~h}$ de tratamento, respectivamente, eficiência de 92,1\% e 94,4\%. Porém, após 192 $\mathrm{h}$ o frasco com $19 \mathrm{~mL}$ reduziu a DQO em $94,4 \%$ e o com $9 \mathrm{~mL} 95,4 \%$, já o controle demonstrou eficácia de 89,2\% em 144 h e 94\% em $192 \mathrm{~h}$. 


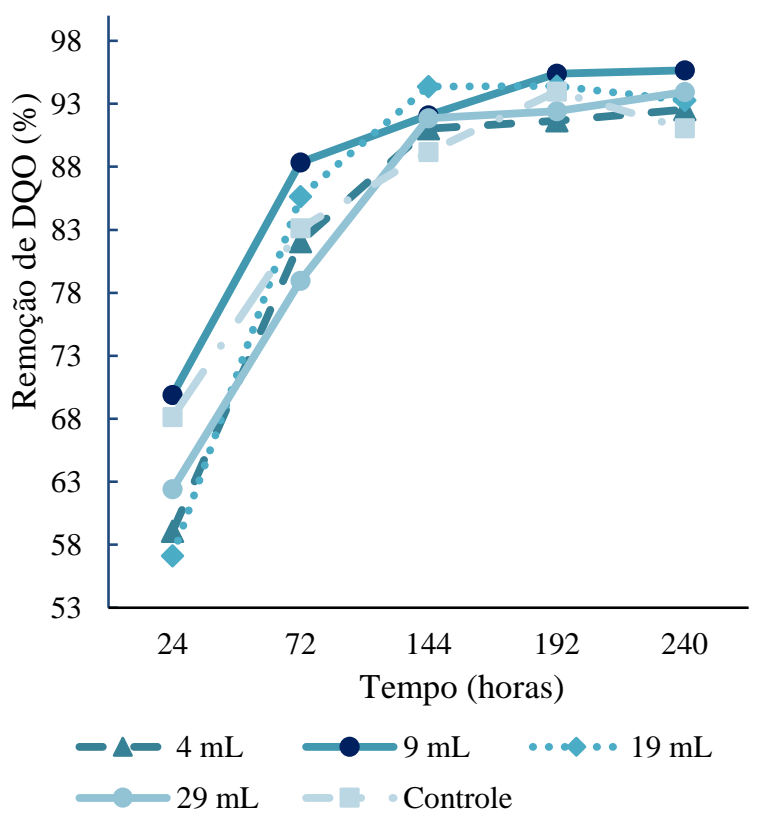

Figura 4. Remoção de DQO nos diferentes ensaios de bioaumentação.

Normalmente o processo da oxidação da amônia a nitrito e posteriormente a nitrato ocorrem pela via biológica e depende do consumo de oxigênio (CANTO et al., 2008; ZOPPAS et al., 2016). Este fenômeno, possivelmente, explicaria a redução dos teores da amônia em todas as amostras, como o demonstrado na Tabela 2.

Entretanto, o frasco controle exibiu maior remoção de amônia, estando acima de $50 \%$ a partir de 72 horas de tratamento. Nos frascos onde adicionou-se o caldo com as colônias de microrganismos, apesar de ocorrer a degradação da amônia e a mesma atingir níveis próximos ao do controle em alguns casos (4 e $9 \mathrm{~mL}$ ), após 240 horas de tratamento, nos demais períodos a taxa de remoção era inferior. Tal fato está, provavelmente, relacionado ao acréscimo de caldo nutriente aos sistemas, já que quanto maior era o volume de caldo adicionado menor era a eficiência da degradação da amônia.

O nitrito, composto intermediário no processo de nitrificação da amônia (ZOPPAS et al., 2016), é instável e rapidamente oxidado a nitrato (CUENCA e REZA, 2013). Por isto, as concentrações de nitrito em amostras, na maioria das vezes, são baixas.

Durante os ensaios de bioaumentação as concentrações de nitrito estiveram abaixo dos teores observados para o esgoto bruto, $0,22 \mathrm{mg} \mathrm{\textrm {L } ^ { - 1 }}$. Estudos apontam que uma concentração limitada de oxigênio no sistema propicia o acúmulo de nitrito (BERNET et al., 2001; ANTILEO et al., 2013). Isto ocorre porque, baixas concentrações de oxigênio dissolvido interferem, de forma mais significativa nas velocidades de crescimento dos microrganismos responsáveis pela oxidação do nitrito do que na velocidade de crescimento dos que oxidam a amônia (ZOPPAS et al., 2016).

Em outras palavras, as bactérias que oxidam a amônia são menos restritivas quanto a presença de oxigênio dissolvido, e a fossa séptica, apesar de ser um ambiente que favoreça o desenvolvimento de microrganismos anaeróbios ou facultativos, apresenta teores significativos de oxigênio dissolvido, 2,55 $\mathrm{mg} \mathrm{L}^{-1}$, possibilitando assim o processo de oxidação da amônia, porém restringindo a etapa de oxidação do nitrito. Deste modo, as informações anteriormente descritas podem justificar os resultados observados para o nitrito.

Ainda com relação ao nitrito, os maiores valores observados para este parâmetro foi no frasco controle. Tal fenômeno, provavelmente, acha-se associado ao processo de degradação da amônia, já que esta última é oxidada a nitrito e posteriormente a nitrato pela via biológica, preferencialmente, aeróbia. 
Tabela 2. Concentração final dos poluentes no esgoto tratado pelo processo de bioaumentação.

\begin{tabular}{|c|c|c|c|c|c|c|c|}
\hline \multirow{2}{*}{$\begin{array}{c}\text { Parâmetro } \\
*\end{array}$} & \multirow{2}{*}{$\begin{array}{c}\text { Esgoto } \\
\text { Bruto }\end{array}$} & \multirow{2}{*}{ Amostra } & \multicolumn{5}{|c|}{ Tempo (horas) } \\
\hline & & & 24 & 72 & 144 & 192 & 240 \\
\hline \multirow{5}{*}{$\begin{array}{l}\text { Amônia } \\
\left(\mathrm{mg} \mathrm{L}^{-1}\right)\end{array}$} & \multirow{5}{*}{20,77} & 4 & $12,94 \pm 3,86$ & $11,18 \pm 5,59$ & $10,78 \pm 1,50$ & $9,64 \pm 1,59$ & $8,55 \pm 1,22$ \\
\hline & & 9 & $13,87 \pm 4,52$ & $13,79 \pm 3,91$ & $11,13 \pm 1,48$ & $10,13 \pm 1,27$ & $8,37 \pm 1,71$ \\
\hline & & 19 & $19,36 \pm 7,89$ & $16,32 \pm 1,96$ & $12,50 \pm 1,44$ & $11,40 \pm 1,53$ & $9,27 \pm 1,56$ \\
\hline & & 29 & $20,79 \pm 7,15$ & $16,74 \pm 1,11$ & $15,38 \pm 1,02$ & $12,85 \pm 1,07$ & $10,75 \pm 1,60$ \\
\hline & & Controle & $12,57 \pm 5,79$ & $9,98 \pm 2,43$ & $9,31 \pm 1,32$ & $8,80 \pm 1,29$ & $8,09 \pm 1,56$ \\
\hline \multirow{5}{*}{$\begin{array}{l}\text { Nitrito } \\
\left(\mathrm{mg} \mathrm{L}^{-1}\right)\end{array}$} & \multirow{5}{*}{0,22} & 4 & $0,193 \pm 0,040$ & $0,067 \pm 0,033$ & $0,023 \pm 0,013$ & $0,048 \pm 0,023$ & $0,055 \pm 0,027$ \\
\hline & & 9 & $0,180 \pm 0,054$ & $0,052 \pm 0,023$ & $0,018 \pm 0,004$ & $0,043 \pm 0,017$ & $0,053 \pm 0,017$ \\
\hline & & 19 & $0,160 \pm 0,057$ & $0,063 \pm 0,029$ & $0,023 \pm 0,008$ & $0,052 \pm 0,023$ & $0,062 \pm 0,022$ \\
\hline & & 29 & $0,138 \pm 0,064$ & $0,070 \pm 0,024$ & $0,020 \pm 0,004$ & $0,047 \pm 0,020$ & $0,060 \pm 0,014$ \\
\hline & & Controle & $0,207 \pm 0,056$ & $0,073 \pm 0,024$ & $0,035 \pm 0,005$ & $0,060 \pm 0,015$ & $0,063 \pm 0,017$ \\
\hline \multirow{5}{*}{$\begin{array}{l}\text { Oxigenação } \\
\left(\mathrm{mg} \mathrm{L}^{-1}\right)\end{array}$} & \multirow{5}{*}{2,55} & 4 & $9,89 \pm 0,55$ & $10,19 \pm 0,22$ & $10,74 \pm 0,41$ & $10,25 \pm 0,23$ & $9,90 \pm 0,01$ \\
\hline & & 9 & $10,14 \pm 0,28$ & $10,26 \pm 0,22$ & $10,54 \pm 0,46$ & $10,27 \pm 0,19$ & $9,90 \pm 0,01$ \\
\hline & & 19 & $10,04 \pm 0,23$ & $10,32 \pm 0,27$ & $10,70 \pm 0,42$ & $10,33 \pm 0,11$ & $10,03 \pm 0,01$ \\
\hline & & 29 & $10,12 \pm 0,24$ & $10,32 \pm 0,30$ & $10,64 \pm 0,46$ & $10,36 \pm 0,09$ & $10,05 \pm 0,01$ \\
\hline & & Controle & $10,10 \pm 0,37$ & $10,37 \pm 0,24$ & $10,76 \pm 0,41$ & $10,35 \pm 0,09$ & $10,00 \pm 0,01$ \\
\hline \multirow{5}{*}{$\mathrm{pH}$} & \multirow{5}{*}{5,91} & 4 & $7,01 \pm 0,23$ & $7,00 \pm 0,28$ & $6,65 \pm 0,35$ & $6,72 \pm 0,26$ & $6,59 \pm 0,15$ \\
\hline & & 9 & $6,96 \pm 0,17$ & $7,14 \pm 0,23$ & $7,05 \pm 0,12$ & $6,92 \pm 0,25$ & $6,79 \pm 0,16$ \\
\hline & & 19 & $6,81 \pm 0,23$ & $7,05 \pm 0,29$ & $7,02 \pm 0,15$ & $6,88 \pm 0,35$ & $6,80 \pm 0,27$ \\
\hline & & 29 & $6,68 \pm 0,17$ & $7,09 \pm 0,33$ & $7,14 \pm 0,23$ & $6,84 \pm 0,42$ & $6,70 \pm 0,24$ \\
\hline & & Controle & $6,79 \pm 0,32$ & $7,00 \pm 0,19$ & $7,11 \pm 0,17$ & $6,68 \pm 0,14$ & $6,60 \pm 0,18$ \\
\hline
\end{tabular}

* Média \pm Desvio Padrão

As taxas de oxigênio foram mantidas próximas a valores de $10 \mathrm{mg} \mathrm{L}^{-1}$. O sistema foi aerado para simular o processo aeróbio e permitir o processo de mistura e oxidação. $\mathrm{O}$ fornecimento deste nos experimentos deu-se por aeração submersa, pois segundo testes realizados por Tose e Lage Filho (2016) os aeradores submersos apresentaram eficiência superior aos aerados superficiais com relação aos processos de mistura e oxigenação do tanque.

Quando em comparação com o esgoto bruto ( $\mathrm{pH}$ 5,91), o $\mathrm{pH}$ das amostras no transcorrer de todo o experimento se manteve em níveis próximos ao neutro $(\mathrm{pH} \mathrm{7,0).} \mathrm{Isto,}$ possivelmente, deve-se ao processo de degradação em si, que leva a decomposição da matéria orgânica, somado ao fato da inserção de oxigênio no meio que contribui com a oxidação e volatilização de compostos. A exemplo deste último é a volatilização do $\mathrm{CO}_{2}$ (DROSTE e GEHR, 2019), subproduto do processo de degradação pela via aeróbia que altera o $\mathrm{pH}$ do meio.

Em suma, com base nos dados do experimento, observou-se que o recipiente onde adicionou-se as colônias de bactérias crescidas em $9 \mathrm{~mL}$ de caldo nutriente demonstrou melhor desempenho na remoção de cor, turbidez e DQO com um tempo de 192 horas de tratamento. Porém, durante a remoção da amônia o frasco controle apresentou melhores resultados. 


\subsubsection{Análise do melhor tratamento de bioaumentação em comparação com o esgoto bruto e o controle}

A Tabela 3 traz em seu escopo o melhor tratamento empregando a técnica de bioaumentação, em comparação ao controle após submissão do esgoto bruto ao mesmo tempo de tratamento.

Em análise a tabela, observa-se que o frasco controle apresentou eficiência superior apenas na remoção do poluente amônia (57,6\%). Para os parâmetros cor, turbidez e DQO o frasco onde adicionou-se $9 \mathrm{~mL}$ de inóculo apresentou melhor desempenho.

A maior concentração de nitrito no frasco controle em comparação ao que recebeu $9 \mathrm{~mL}$ de caldo nutriente, deve-se, provavelmente, ao processo de oxidação da amônia. Contudo, tal situação já foi explanada no item anterior.

De maneira geral, em análise dos resultados obtidos nos ensaios laboratoriais e tendo por base o preceito que quanto maior, ou mais eficiente, for a remoção de poluentes de uma água residuária menor será o impacto negativo desta sobre o meio ambiente, é possível afirmar que o uso da técnica de bioaumentação melhorou a remoção de determinados poluentes presentes na água residuária estudada.
Resultados positivos com o uso da técnica de bioaumentação também foram observados por Nzila et al. (2016) durante um trabalho de revisão a respeito do emprego desta técnica no tratamento de águas residuárias industriais. Mehrotra et al. (2019) mostraram em seus experimentos que a biodegradação de águas residuais domésticas pode ser alcançada de maneira rápida e melhor pela incorporação de consórcio de bactérias.

Em experiências com águas residuais municipais, Hesnawi et al. (2014) constaram que os reatores operados com bioaumentação apresentaram resultados melhores que $\mathrm{O}$ controle (operado sem bioaumentação). Além disto, observaram que o reator inoculado com cepas selecionadas combinadas possuíam eficácia relativamente maior quando comparado aos reatores inoculados com uma única cepa.

Por fim, em ensaios laboratoriais Chen et al. (2014) verificaram que o emprego da técnica de bioaumentação (flora mista) no tratamento de águas residuárias de celulose não apenas poderia aumentar a eficiência da remoção de DQO, mas também melhorar a capacidade de descoloração.

Tabela 3. Comparação entre melhor tratamento de bioaumentação e o controle.

\begin{tabular}{lccc}
\hline Parâmetro* $^{*}$ & Esgoto Bruto & $\begin{array}{c}\text { Controle } \\
(\mathbf{1 9 2} \text { h) }\end{array}$ & $\begin{array}{c}\text { Amostra 9 mL } \\
(\mathbf{1 9 2} \text { h) }\end{array}$ \\
\hline Amônia $\left(\mathrm{mg} \mathrm{L}^{-1}\right)$ & $20,77 \pm 10,71$ & $8,80 \pm 1,29$ & $10,13 \pm 1,27$ \\
Cor aparente $\left(\mathrm{mg} \mathrm{Pt} \mathrm{Co} \mathrm{L}^{-1}\right)$ & $2112,22 \pm 779,39$ & $221,80 \pm 39,12$ & $106,00 \pm 41,38$ \\
DQO $\left(\mathrm{mg} \mathrm{L}^{-1}\right)$ & $585,63 \pm 31,05$ & $35,28 \pm 13,02$ & $26,92 \pm 11,11$ \\
Oxigênio dissolvido $\left(\mathrm{mg} \mathrm{L}^{-1}\right)$ & $2,55 \pm 0,58$ & $10,35 \pm 0,09$ & $10,27 \pm 0,19$ \\
Nitrito $\left(\mathrm{mg} \mathrm{L}^{-1}\right)$ & $0,22 \pm 0,08$ & $0,060 \pm 0,015$ & $0,043 \pm 0,017$ \\
pH & $5,91 \pm 0,17$ & $6,68 \pm 0,14$ & $6,92 \pm 0,25$ \\
Turbidez (NTU) & $213,60 \pm 75,94$ & $9,13 \pm 4,78$ & $6,45 \pm 3,69$ \\
\hline
\end{tabular}

* Média \pm Desvio Padrão 


\section{CONSIDERAÇões FINAIS}

O emprego da bioaumentação pode contribuir para a redução da carga poluidora de determinado resíduo, principalmente, os que são de difícil degradação. Esta técnica pode ser ainda valiosa para dar o start em novas estações de tratamento, além de reduzir o tempo e o custo de tratamento em estações que recebem volumes consideráveis de águas residuárias.

Deste modo, com base nos resultados aferidos com este estudo e das informações disponíveis na literatura, é possível afirmar que a técnica de bioaumentação é eficiente no processo de remoção de determinados compostos. E a utilização de microrganismos nativos do poluente contribuem para otimizar o processo.

Contudo, a fim de verificar as afirmações anteriores, sugere-se como trabalhos futuros realizar a identificação da maioria das bactérias presentes no esgoto da universidade, selecionar as espécies que demonstrarem melhor crescimento em laboratório e inoculá-las ao esgoto de maneira separada e em consórcio e, posteriormente, realizar uma comparação com os resultados obtidos neste experimento.

\section{Agradecimentos}

Os autores agradecem a Universidade do Oeste de Santa Catariana - Unoesc Videira, e ao Programa de Bolsas Universitárias de Santa Catarina (UNIEDU). E aproveitam para estender os agradecimentos aos avaliadores deste artigo pelas dicas de melhorias.

\section{REFERÊNCIAS}

AMARAL, AC; STEINMETZ, RLR; KUNZ, A. O processo de biogestão. In: KUNZ, A.; STEINMETZ, RLR; AMARAL, AC(ed.). Fundamentos da digestão anaeróbia, purificação do biogás, uso e tratamento do digestato. Concórdia, SC: SBERA, Embrapa, 2019. p. 13-26.

ANTILEO, C; MEDINA, H; BORNHARDT, C; MUÑOZ, C; JARAMILLO, F; PROAL, J. Actuators monitoring system for real-time control of nitrification-denitrification via nitrite on long term operation. Chemical Engineering Journal, v. 223, p. 467-478, maio. 2013.

APHA-AWWA-WEF. Standard methods for the examination of water and wastewater. 22 th. Baltimore (USA): APHA, 2012. $1 \mathrm{v}$.

BERNET, N; DANGCONG, P; DELGENÈS, $\mathrm{J}$; MOLETTAS, R. Nitrification at low oxygen concentration in biofilm reactor. Journal of Environmental Engineering, v. 127, n. 1, p. 1-6, mar. 2001.

CANTO, CSA; RATUSZNEI, SM; RODRIGUES, JAD; ZAIAT, M; FORESTI, E. Effect of ammonia load on efficiency of nitrogen removal in an SBBR with liquidphase circulation. Brazilian Journal of Chemical Engineering, v. 25, n. 2, p. 275289, abr./jun. 2008.

CHEN, H; YANG, G; CHEN, J; LIU, Y. Enhanced biodegradability of aerobic sludgen by bioaugmentation for pulping effluent treatment. BioResources, v. 9, n. 2, p. 21432153, 2014.

COLARES, CJG; SANDRI, D. Eficiência do tratamento de esgoto com tanques sépticos seguidos de leitos cultivados com diferentes meios de suporte. Revista Ambiente \& Água, v. 8, n.1, p. 172-185, 2013.

CUENCA, MA; REZA, M; CUBEN. A novel bioreactor for the removal of nutrients. In: COCA-PRADOS, J; GUTIÉRREZCERVELLÓ, G. Economic sustainability and environmental protection in Mediterranean countries through clean 
manufacturing methods. The Netherlands: Springer, 2011. p. 63-92.

DROSTE, R; GEHR, R. Theory and practice of water and wastewater treatment. 2 . ed. Hoboken: Wiley, 2019.

HERRERO, M; STUCKEY, DC. Bioaugmentation and its application in wastewater treatment: a review. Chemosphere, v. 140, p. 119-128, dez. 2015.

HESNAWI, R; DAHMANI, K; ALSWAYAH, A; MOHAMED, S; MOHAMMED, SA. Biodegradation of municipal wastewater with local and commercial bacteria. Procedia Engineering, v. 70, p. 810-814, 2014.

JORDÃO, EP; PESSÔA, CA. Tratamento de esgotos domésticos. 4. ed. Rio de Janeiro: ABES, 2005. xxvi, 906 p.

KOREN, H; BISESI, M. Handbook of environmental health: pollutant interactions in air, water, and soil. Boca Raton, Lewis Publishers, 2003. 904 p. v. 2.

MANAGO, BL; VIDAL, CMS; SOUZA, JB; NEVES, LC; MARTINS, KG. Dissolved air flotation for fiber removal from clear water. Floresta e Ambiente, v. 25, n. 2, p. 1-10, fev. 2018 .

MANYUCHI, MM; MBOHWA, C; MUZENDA, E. Resource recovery from municipal sewage plants: an energy-waternutrients nexus for developing countries. Boca Raton: CRC Press Taylor \& Francis Group, 2019. 146 p.

MEHROTRA, T; SRIVASTAVA, A; RAO, RP; SINGH, R. A Novel Immobilized bacterial consortium bioaugmented in a bioreactor for sustainable wastewater treatment. Journal of Pure and Applied Microbiology, v. 13, n. 1, p. 371-383, mar. 2019.
NOWACKI, CCB; RANGEL, MBA Química Ambiental: conceitos, processos e estudo dos impactos ao meio ambiente. 1. ed. São Paulo: Érica, 2014. 136 p.

NUVOLARI, A. Esgoto sanitário: coleta, transporte, tratamento e reúso agrícola. 2. ed., rev., atual. e ampl. São Paulo: Edgard Blücher, 2011. $565 \mathrm{p}$.

NZILA, A; RAZZAK, SA; ZHU, J. Bioaugmentation: an emerging strategy of industrial wastewater treatment for reuse and discharge. International Journal of Environmental Research and Public Health, v. 13, n. 9, p. 1-20, ago. 2016.

PEIXOTO, FS; LIMA, BG; BARROS, ARM; SILVA FILHO, HA; SANTOS, EVM. Importância da Caracterização de Esgotos Gerados em Instituições de Ensino - Estudo de Caso - IFCE, Campus Limoeiro do Norte. In: VII CONNEPI CONGRESSO NORTE NORDESTE DE PESQUISA E INOVAÇÃO, 2012, Palmas - TO.

SHAH, M. Bio-Augmentation: a fantabulous technology in waste water treatment. International Journal of Waste Resources, v. 7, n. 1, p. 1-3, 2017.

SKINDER, BM; UQAB, B; GANAI, BA. Bioremediarion: a sustainable and emerging tool for restoration of polluted aquatic ecosystem. In: QADRI, H; BHAT, RA; MEHMOOD, MA; DAR GH (ed). Fresh water pollution dynamics and remediation. Singapore: Springer Singapore Pte Ltd., 2020. p. 143-166.

SOUSA, MU. Análise físico-química e microbiológica do esgoto de uma universidade pública com proposta de tratamento biológico para reuso na própria instituição. In: CONGRESSO NACIONAL DE PESQUISA E ENSINO EM CIÊNCIAS, 2016, Campina Grande - PB. 
SPEIGHT, JG; EL-GENDY, NS. Introduction to petroleum biotechnology. Cambridge: Gulf Professional Publishing, 2018. 566 p.

SZAJA, A; LAGÓD, G; JAROMIN-GLÉN, K; MONTUSIEWICZ, A. The effect of bioaugmentation with archaea on the oxygen uptake rate in a sequencing batch reactor. Water, v. 10, n. 5, p. 1-11, 2018.

TCHOBANOGLOUS, G; STENSEL, HD; TSUCHIHASHI, R; BURTON, F. Wastewater engineering: treatment and reuse. 5. ed. New York: The McGraw-Hill, 2013. 2048 p.

TONETTI, AL; CORAUCCI FILHO, B; NICOLAU, CE; BARBOSA, M; TONON, D. Tratamento de esgoto e produção de água de reuso com o emprego de filtros de areia. Engenharia Sanitária e Ambiental, v. 17, n.3, p. 287-294, jul./set. 2012.

TOPARE, NS; ATTAR, SJ; MANFE, MM Sewage/wasterwater treatment technologies: a review. Scientific Reviews \& Chemical Communication, v. 1, n. 1, p. 18-24, 2011.

TORTORA, GJ; FUNKE, BR; CASE, CL. Microbiologia. 2. ed. Porto Alegre: Artmed, 2017. $935 \mathrm{p}$

TOSI, DA; LAGE FILHO, FA. Comparação de eficiência no tratamento de esgotos domésticos: aeração superficial $X$ aeração submersa. Revista Acadêmica, v. 3, n. 12, p. 1-13, out./dez. 2016.

TURCI, LFR; MENEZES, LCC; MOURA, RB. Caracterização do efluente gerado no campus Poços de Caldas da Universidade Federal de Alfenas. In: CONGRESSO ABES FENASAN, 2017, São Paulo.

VON SPERLING, M. Introdução à qualidade das águas e ao tratamento de esgotos. 3. ed. Belo Horizonte: Desa, UFMG, 2005. 452 p.

ZHANG， Q; YANG， G; ZHANG， L; ZHANG, Z; TIAN, G; JIN, R. Bioaugmentation as a useful strategy for performance enhancement in biological wastewater treatment undergoing different stresses: application and mechanisms. Critical Reviews in Environmental Science and Technology, v. 47, n. 19, p. 1877-1899, 2017.

ZOPPAS, FM; BERNARDES, AM; MENEGUZZI, A. Parâmetros operacionais na remoção biológica de nitrogênio de águas por nitrificação e desnitrificação simultânea. Engenharia Sanitária Ambiental, v. 21, n. 1, p. 29-42, jan./mar. 2016. 